\title{
Moviéndose CON LA MENTE Y EL CUERPO: ENTENDIENDO LA DIFERENCIA ENTRE PLANEAR Y REALIZAR RUTAS EN EL ESPACIO CONSTRUIDO
}

Rodrigo Mora ${ }^{\star}$

\begin{abstract}
RESUMEN
Este escrito estudió hasta qué punto las personas, al momento de ir de un punto $A$ a uno $B$, realizan las rutas planeadas previamente, y si éstas son idénticas para ir que para volver. Se pidió a 42 participantes estudiar un plano del campus principal de la UTFSM, y trazar las rutas que eligirian para ir entre dos pares de puntos. Una vez terminada esta tarea, los participantes debieron recorrer en la realidad las rutas trazadas. Los resultados mostraron que las rutas trazadas en papel no necesariamente coincidieron con las rutas realizadas en terreno, y que, en la mayoría de los casos, las rutas $A-B / B-A$ y $X-Y / Y-X$ no fueron simétricas. Se concluye sugiriendo que los criterios de elección de ruta cambian porque el razonamiento espacial de las personas es inherentemente situado, esto es, moldeado por el entorno en que se lleva a cabo.

Palabras claves: distancia, cognición espacial, cognición situada.

\section{MOVING WITH THE MIND AND THE BODY: EXECUTING ROUTES IN THE BUILT ENVIRONMENT} UNDERSTANDING THE GAP BETWEEN PLANNING AND
\end{abstract}

\begin{abstract}
This paper is concerned with route behavior in people. Specifically, it aims to better understand whether people follow routes planned in advance, and whether these routes are identical irrespective of the direction of travel. A total of 42 participants (27 belonging to UTFSM and 15 from outside) were asked to trace routed between a pair of points in a map of the campus. After that, they were brought to these places and asked to physically navigate these routes. The main results showed that planned routes were not necessarily employed in real-world scenarios, and that people often changed routes depending on the direction of travel. These results suggest that route choice criteria are somehow dependant on contextual information.
\end{abstract}

Keywords: distance; spatial cognition; situated cognition.

^Arquitecto. Departamento de Arquitectura, Universidad Técnica Federico Santa María. Avenida España 1680, Valparaíso, Chile.

Correo electrónico: rodrigo.mora@usm.cl 


\section{1.- INTRODUCCIÓN}

Desde hace más de cincuenta años la cognición espacial, sub disciplina surgida del cruce entre la sicología y la geografía, viene estudiando cómo la gente se mueve y orienta en el espacio. Algunas de las preguntas fundamentales que esta ciencia aspira a responder son: ¿Cómo se forman los "mapas cognitivos"representaciones abstractas del espacio- en mente de las personas?, ¿Cómo es percibida la distancia? o por último, ¿Cuáles son los criterios definidos por las personas al planificar, y luego ejecutar, una ruta?

Este escrito se centra en la última de estas preguntas, la relacionada con la planificación y ejecución de rutas. Esto por dos razones. La primera es todos nosotros, a diario, debemos definir rutas para ir a distintos lugares, como el supermercado, a nuestros trabajos o el paradero del autobús. Definir mentalmente rutas y ejecutarlas parece ser un problema corriente, doméstico. La segunda razón es que, a pesar de este carácter, los criterios de definición de rutas no son transparentes sino que dependen de una serie de factores, de los cuales el principal es la percepción de distancia. Por ello, entender cómo las personas definen un asunto aparentemente simple, como el definir un camino entre dos puntos, ayuda a entender un problema cognitivamente más complejo, como el relacionado con la distancia.

\section{2.- Trabajo Previo}

La gran mayoría de las investigaciones sobre la elección de rutas se centran en cómo las personas perciben la distancia entre dos o más puntos. Al respecto, se ha sostenido que la distancia entre dos puntos puede ser medida de varias formas. Una, quizás la más simple, es la Euclidiana, y dice relación con la magnitud en metros, kilómetros o cualquier otra medida, de una línea recta trazada entre dos puntos. También existe lo que se conoce como distancia "de ruta", o distancia del o los caminos que unen dos puntos. La distancia topológica, por otro lado, dice relación con la cantidad de cambios de dirección que deben hacerse entre dos locaciones (MONTELLO, 1991). Por último, la distancia entre dos puntos puede ser medida en minutos, costo monetario o esfuerzo físico.

Varios estudios han mostrado que la distancia es un concepto multidimensional. Por ejemplo, investigaciones realizadas en los ochenta por Sadalla y colegas mostraron que una calle con más intersecciones es percibida como más larga que una calle con menos intersecciones (SADALLA; STAPLIN, 1980), y que lo mismo ocurre cuando una calle tiene muchos cambios de dirección (SADALLA; MAGEL, 1980). Asimismo, se demostró que el grado de familiaridad de las nombres de las calles puede influir en la percepción de la distancia (SADALLA; STAPLIN; BURROUGHS, 1979), vale decir, que el atravesar calles con nombres poco comunes hace que la distancia percibida de la calle por la que se circula sea más larga que si éstas avenidas tuvieses calles más familiares.

Experimentos realizados en situaciones menos experimentales (en ciudades), han mostrado que las personas tienden a subestimar las distancias de más de 7 kilómetros, y a sobreestimar las de menos de 7 kilómetros (CANTER; 
KAGG, 1975; GOLLEDGE; STIMSON, 1997). En la misma línea, se ha mostrado que la percepción de distancia varía a lo largo del tiempo, es decir, que aquellos que han permanecido mucho tiempo en un lugar perciben la distancia entre dos puntos en forma diferente que aquellos que han permanecido poco tiempo. Por ejemplo, un experimento realizado en estudiantes norteamericanos (CROMPTON, 2006), mostró que una milla de distancia era estimada un $24 \%$ más larga por estudiantes de primer año de la Universidad, un 33\% más larga por estudiantes de segundo año, y un $45 \%$ más extensa por los de tercer año. Finalmente, varios estudios han mostrado que la percepción de distancia puede verse afectada por eventos como la construcción de un puente (ANTES; MCBRIDE; COLLINS, 1988), por el oficio de la persona que hace la estimación (PERUCH; GIRAUDO; GÄRLING, 1989), o por el grado de actividad física de la persona (KIRTLAND et al., 2003; MCCORMACK et al., 2008).

Lo anterior ha impulsado a varios autores a intentar establecer ciertos principios generales (o heurísticas), sobre la forma en que la distancia es entendida por las personas cuando éstas deben ir de un punto A a un punto B. Al respecto, se ha sostenido que los humanos siguen la "regla del segmento inicial" (ISS según sus siglas en inglés), que sugiere que, enfrentadas a dos o más rutas entre dos puntos, las personas elegirán aquella con el segmento inicial más largo pues percibirán este camino como el más corto (BAILENSON; SHUM; UTTAL, 2000). Otros autores (CONROY-DALTON, 2003; HOCHMAIR, 2005), han refinado esta idea, sugiriendo que esta regla será seguida siempre y cuando el ángulo de desviación del segmento inicial más largo sea menor que los demás segmentos iniciales respecto del destino final de la persona.

La preocupación por la idea de distancia, y cómo este concepto afecta las decisiones espaciales de las personas al momento de elegir rutas, llevó al norteamericano Reginald Golledge, uno de los principales expertos en cognición espacial, a realizar en 1995 un interesante experimento. En él se pidió a 32 estudiantes universitarios (16 hombres y 16 mujeres), que dibujaran en un plano la ruta que harían entre cuatro orígenes y cuatro destinaciones. Todas las rutas tenían el mismo largo y pertenecían a uno de los patios del campus, aunque eran difícilmente reconocibles por los estudiantes en el plano. Una vez terminada esta tarea, Golledge llevó físicamente a los estudiantes a estos lugares y les solicitó que caminaran primero de $\mathrm{A}$ hasta $\mathrm{B}$ y luego desde $\mathrm{B}$ hasta $\mathrm{A}$, y que después hicieran lo mismo con los puntos $\mathrm{X}$ e Y.

Tres eran quizás las hipótesis más interesantes de Golledge. La primera sostenía que el criterio de selección de ruta sería similar en la condición de laboratorio - cuando los participantes debían trazar en el papel los caminos entre puntos- y en la realidad. La segunda planteaba que los caminos de ida y de vuelta serían, en su gran mayoría, idénticos, vale decir, que para ir desde A hasta B los participantes elegirían el mismo camino que para ir de B a A. Por último, Golledge sostenía que los criterios que prevalecerían en la de ruta sería el del "camino más corto" y el del "menor tiempo". 
Para sorpresa del científico, ni una de estas ideas idea no se cumplió en la mayoría de los casos. Eso porque una minoría de las personas hicieron en terreno la ruta que dijeron harían en el papel, y porque las rutas empleadas para ir no fueron las mismas que las usadas para volver. Más aún, los criterios considerados como dominantes para la elección de rutas (el del camino más corto y el del menor tiempo), no necesariamente fueron los empleados por los participantes.

Intrigado por estos resultados, Golledge sugirió que probablemente las interpretaron de forma diferente la distancia entre los puntos según su posición en el espacio, lo que a su vez incidía en el criterio elegido para selecciona el camino entre las cuatro rutas.

Intentando ahondar en estas ideas, acá se expone un experimento similar al realizado por Golledge, pero que en vez de usar sólo estudiantes universitarios familiarizados con el campus en el que debían imaginar y caminar dos rutas, acá se incluyó a personas de fuera de la Universidad. Esto porque varios estudios indican que el grado de familiaridad con el entorno puede afectar tanto los criterios de planificación de una ruta entre dos puntos (LEISER; ZIBERSHATZ, 1989), como la forma en que esta planificación es realizada en la práctica (GOLLEDGE et al., 1985). Se pensaba entonces que las personas ajenas a la universidad tendrían una pauta de comportamiento diferente a los estudiantes, que a diario recorren el campus.

\section{MÉTodo}

\section{Escenarios}

El escenario elegido para este experimento fue uno de los patios (Patio de los Ceibos) del campus central de la Universidad Técnica Federico Santa María, en Valparaiso, Chile. Este campus alberga a cerca de 5,000 estudiantes, la mayoría de los cuales cursan carreras ligadas a la ingeniería o ciencias exactas. El complejo, de gran valor arquitectónico, se estructura en torno a patios dispuestos en distintos niveles, los que deben ser atravesados constantemente por los alumnos para ir o venir de sus laboratorios y salas de clases.

El patio en cuestión tiene una forma casi cuadrada de casi $100 \mathrm{~m}$ de arista (figura 1). Tres de los lados de este cuadrado corresponden a edificios que albergan salas de clases y oficinas (los departamentos de física, industrias e informática), mientras el cuarto conduce, después de atravesar una desnivel de aproximadamente 1.5 metros, a otro patio donde es posible observar la bahía de Valparaiso. La conformación interior del Patio de los Ceibos incluye un espacio central, rodeado por una serie de espacios de pasto y arbustos bajos, además de varios ceibos (de ahí su nombre) de gran altura y frondosidad. 


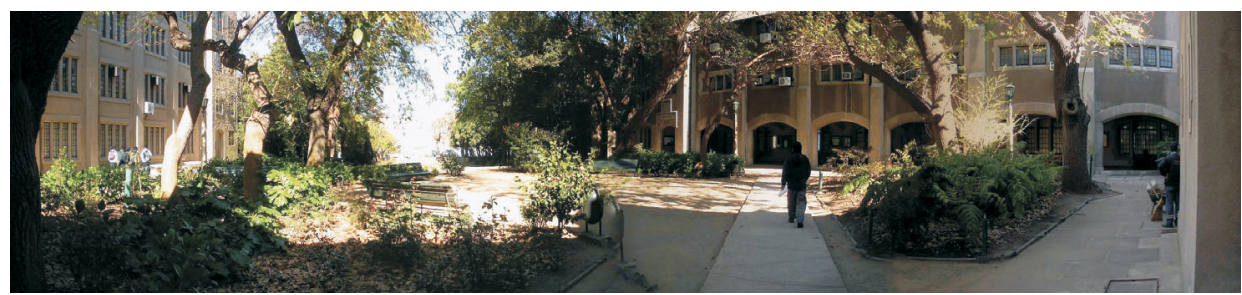

Figura 1: Patio de los Ceibos

\section{Materiales y Diseño}

Los participantes recibieron una hoja formato A4 con un plano abstracto del patio de los Ceibos, que mostraba su subdivisión interior de éste y los bordes que lo rodean. Con el objeto de que los participantes no pudieran fácilmente distinguir de qué área se trataba, la planta se simplificó fuertemente los contornos del patio, al tiempo que se giró en $90^{\circ}$ el plano respecto de la posición los participantes. Todos los participantes debieron completar la tarea en la misma posición. La figura 2 muestra estas operaciones.

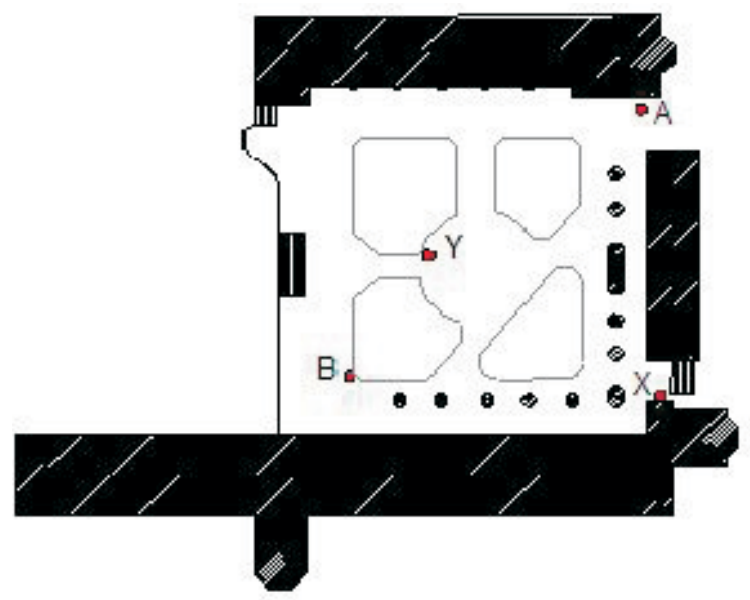

Figura 2: plano mostrado a los participantes.

En el plano se indicaban cuatro puntos: A, B, X e Y. Los participantes tuvieron que marcar con un lápiz la ruta que harían si tuvieran que ir de A hasta $\mathrm{B}$, y luego de $\mathrm{X}$ a $\mathrm{Y}$. Se escogieron estos puntos atendiendo al hecho de que el tramo A-B era más largo pero aparentemente más simple, pues existía una senda diagonal que permitía unir los puntos, mientras el tramo X-Y era más corto pero más complejo, pues implicaba vadear un sector de césped. Sin embargo, en ambos casos los puntos de origen y destino eran mutuamente visibles y tenían más de una forma de ser unidos. 


\section{Participantes y Procedimiento}

42 (26 hombres,16 mujeres ) personas participaron voluntariamente en este experimento. De ellas, 27 eran alumnos de la Universidad, y las 15 restantes eran personas que nunca habían estado en el campus. La edad promedio fue de 25.6 años (17 mínima, 57 máxima, Desviación Estándar $=7.58$ ).

En la primera fase del experimento, las personas fueron llevadas a una sala sin ventanas alejada del Patio de los Ceibos. Una vez ahí, debieron sentarse en una ubicación previamente establecida, al tiempo que se les daba el plano en cuestión. Debieron escuchar entonces la siguiente instrucción:

Este es un experimento sobre cómo nos movemos en un edificio. Por favor trace en el siguiente plano cual sería la ruta que usted haría para ir del punto $\mathrm{A}$ hasta el punto $\mathrm{B}$ y luego desde el punto B al punto A. Una vez terminada esta tarea, repita el mismo procedimiento para los puntos $\mathrm{X}$ e $\mathrm{Y}$.

Las personas tuvieron todo el tiempo a su disposición para completar la tarea. Sin embargo, el tiempo que demoraban en hacerlo era registrado discretamente por uno de los encargados del experimento. Una vez terminada la fase de papel, los participantes debieron responder una pequeña encuesta donde se les pidió evaluaran su sentido de orientación en una de las cuatro categorías : muy bueno, bueno, regular y malo. Debieron demás informar cuantos años habían estudiado en la Universidad (en el caso de que fueran estudiantes de ésta).

En la segunda fase del experimento, los participantes fueron llevados individualmente por los encuestadores hasta el Patio de los Ceibos en un horario en que éste se encontraba con muy poca gente. Una vez allí, fueron llevados hasta el punto $\mathrm{A}$, donde les fue indicado llegaran hasta el punto $\mathrm{B}$, donde otro encuestador les esperaba señalándoles con la mano. Al llegar al tal lugar, cada participante debía retornar al punto $\mathrm{A}$. El mismo procedimiento se uso entre los puntos $\mathrm{X}$ e $\mathrm{Y}$. Tal como se hizo en la fase anterior, un tercer encuestador registraba discretamente en un plano el camino realizado por cada participante.

\section{3.- Resultados}

\section{Análisis estadístico}

Primero, se estudió si el sexo de los participantes tenía relación con sentido de orientación que éstos dijeron poseer. Una prueba se Chi-cuadrado no detectó asociación entre estas variables (Chi-cuadrado .135 p>0.05), aunque los hombres fueron levemente más confiados que las mujeres. Luego se midió si los participantes pertenecientes a la UTFSM mostraban un nivel diferente de confianza que los que no lo eran. Acá si se detectó diferencias significativas (Chi-cuadrado 24.2, p $>0.05$ ), pues mientras el 100\% de los estudiantes de la UTFSM dijeron tener un sentido de orientación bueno o muy bueno $(\mathrm{N}=27)$, sólo un $33 \%(\mathrm{~N}=5)$ de los invitados sostuvo lo mismo. Del 66\% restante (10 personas), 8 dijeron tener un sentido 
de orientación malo y 2 uno regular, lo que muestra que el hecho de exponerse a un medio que no conocían hizo que la mayoría de los invitados se sintiera inseguro. No hubo diferencias respecto del tiempo que se tomaron hombres y mujeres $(\mathrm{t}$ $(42)=-2.76 ; \mathrm{p}>0.05$ ), o participantes pertenecientes o no a la UTFSM (ANOVA (F: $2.08 \mathrm{p}>0.05$ ). La edad de los participantes tampoco importantemente estuvo asociada al tiempo demorado en contestar las preguntas (Pearson r: 0.319).

Respecto del grado de coincidencia entre lo trazado en el plano y lo realizado en la realidad, no se detectó una influencia de género, vale decir, ni mujeres ni hombres tuvieron diferencias significativas respecto de lo que trazaron en papel versus lo que hicieron en la realidad. ${ }^{1}$ Asimismo, no encontró relación entre los que realizaron la misma ruta al ir de $\mathrm{A}$ a $\mathrm{B}$ que los de $\mathrm{B}$ a $\mathrm{A}, \mathrm{y}$ los que hicieron los mismo en el caso de los puntos X-Y (Chi-cuadrado 5.63, $\mathrm{p}>0.05$ ).

\section{Análisis de las respuestas dadas por los participantes}

Respecto del grado de coincidencia entre el camino planeado en el mapa y el camino efectuado en la realidad, se detectó que para la ruta A-B, solo el $21.4 \%$ de los encuestados realizó en el terreno el camino trazado en el plano. Este porcentaje subió para el caso de la ruta X-Y, pues el 52.4\% de los participantes realizó en terreno el camino que dibujo en el plano. Pero no sólo hubo una discrepancia entre las rutas planeadas versus las rutas realizadas, sino también hubo una diferencia importante entre los caminos de ida y vuelta realizados por las personas. Mientras en la ruta A-B el 54.8\% de los encuestados realizó el mismo camino de ida que de vuelta, en la ruta X-Y el 69\% tuvo ese comportamiento.

La figura 3 muestra las rutas planeadas y las realizadas por todos los participantes. Acá se puede apreciar que en el caso del par X-Y, la casi totalidad de los participantes circunnavegaron el espacio de pasto que separaba los puntos de origen y destinación, cambiando de dirección dos a tres veces en cada trayecto. A diferencia de este patrón, la mayoría de los que debieron unir a pie los puntos A y $\mathrm{B}$ se movieron por el interior, realizando una trayectoria quebrada de tres o más cambios de dirección. Curiosamente, ni una persona realizó la ruta topológicamente más simple pero métricamente mas demandante que consistía en recorrer dos aristas del cuadrado del patio.

La figura 4 muestra los trayectos planeados y realizados para las rutas A-B y X-Y, por quienes pertenecían y no pertenecían a la Universidad. Tal como se puede apreciar gráficamente, no existieron mayores diferencias en la forma en que ambos grupos primero planificaron, y luego ejecutaron, las dos rutas. En efecto, una prueba de chi-cuadrado no detectó diferencias en este sentido ni para la ruta X-Y (Chi-cuadrado .933, p>0.05), ni para la A-B (Chicuadrado 4.32, $\mathrm{p}>0.05$ ). Tampoco se encontraron diferencias en cuanto al comportamiento de las personas en terreno, vale decir, al momento de moverse físicamente entre los puntos X-Y y A-B (Chi-cuadrado 2.69, p >0.05 para la ruta $X-Y$ y $0.853, p>0.05$ para la ruta $A-B)$. En definitiva, los que no eran de la 
Universidad no tuvieron un comportamiento diferente de los que sí lo eran ni en términos de las forma en que planificaron sus movimientos (al trazar en el papel las rutas), ni en la forma en que se desplazaron.

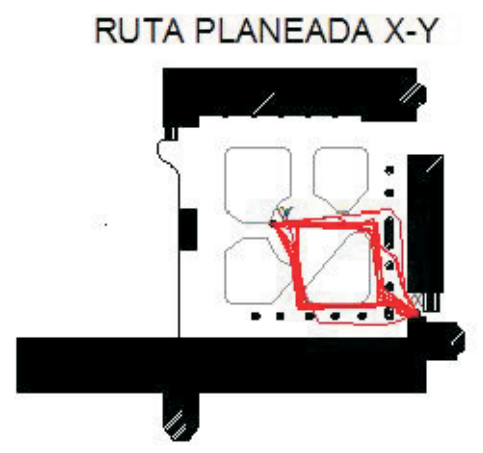

RUTA EJECUTADA X-Y

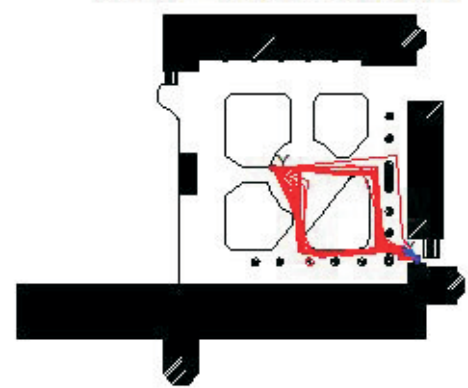

RUTA PLANEADA A-B

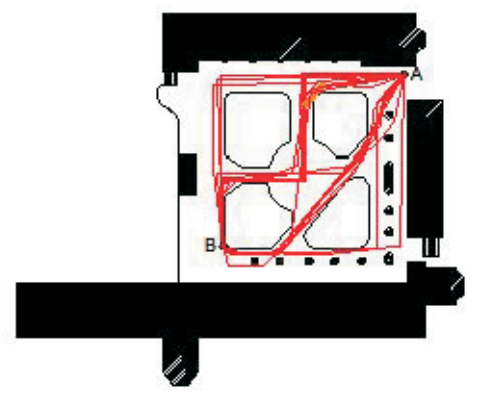

RUTA EJECUTADA A-B

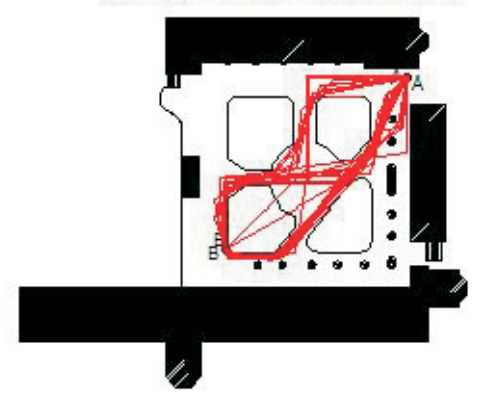

Figura 3: rutas planeadas y realizadas por las personas
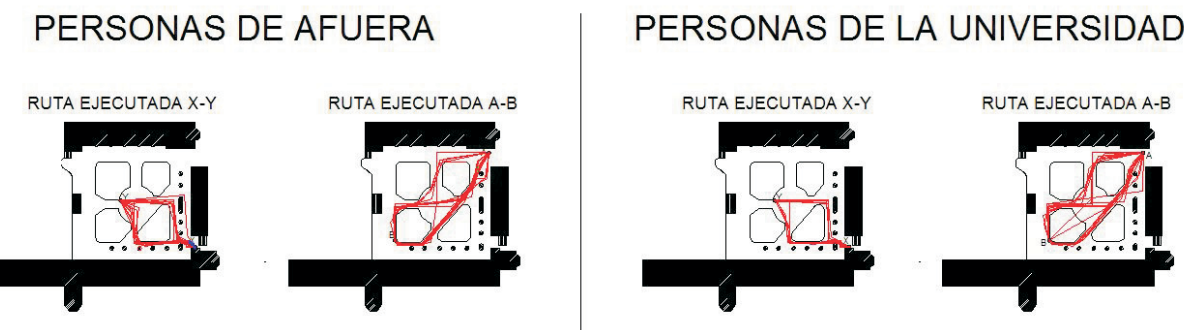

Figura 4: rutas planeadas y realizadas por las personas

\section{4.- Discusión}

A nivel general, los resultados obtenidos por este experimento son coincidentes con los reportados por el experimento de Golledge de 1995, en el sentido de que las rutas definidas por las personas en un mapa no parecen guardar necesariamente una relación con las rutas realizadas in situ por ellas, y que los caminos que la gente toma para ir que para volver de un lugar no necesariamente 
son los mismos. Más aún, este experimento mostró que estas conductas parecen no tener relación del grado de familiaridad de las personas con el medio, pues los que no pertenecían a la Universidad tuvieron un patrón de comportamiento similar a los que sí pertenecían a ella.

El hecho que ningún participante haya realizado trayectorias topológicamente más simples (es decir, con menos quiebres), pero métricamente más demandantes (es decir, más largas), sugiere que las personas tienden a "seguir su nariz" (CONROY-DALTON, 2003), esto es, a preservar trayectorias continuas o semi-continuas al moverse en el espacio. Esto parece concordante con recientes investigaciones en el área (PEPONIS; ZIMRING; YOON, 1990; HILLIER; IIDA, 2005; JANZEN et al., 2000), que apuntan a la existencia ciertas pautas de navegación universales en las personas, cuyo origen estaría relacionado con la propia anatomía humana (TVERSKY, 2003; KLIPPEL; MONTELLO, 2004). En este sentido, se ha sugerido que el eje atrás/adelante, condicionaría en gran parte la forma de movimiento humano, alentando la realización de trayectorias sinuosas

Desde otro punto de vista meta- teórico, los resultados de este experimento parecen apoyar algunas teorías contemporáneas de la cognición, que sugieren que la acción humana esta inherentemente "situada", esto es, que es definida en buena medida por las condiciones medioambientales donde esta se lleva a cabo. Al respecto, se ha propuesto que la acción humana es el resultado de un proceso interdependiente y recursivo entre la mente, el cuerpo y el medio ambiente (VARELA; THOMPSON; ROSCH, 2003; NOË, 2004; THOMPSON, 2007). Más aún, el desarrollo de las teorías cognitivas de tipo "embodied" (CLARK, 1997, 2008), según las cuales el funcionamiento de la mente humana no consiste solamente en manipular información simbólica, sino en responder a las estimulaciones que el propio cuerpo proporciona en su relación con un medio ambiente cambiante, ofrecen un marco explicativo seductor para entendimiento de la acción humana, especialmente la relacionada con la acción espacial de las personas.

Lo anterior pone un signo de interrogación acerca de la validez de heurísticas explicativas generales sobre el movimiento humano en el espacio, especialmente el movimiento de menor escala como el estudiado en este experimento. Esto porque este movimiento está fuertemente condicionado por aspectos contextuales, por lo que su predicción no es un asunto fácil. Investigaciones futuras debieran explorar estos y otros temas.

\section{Nota}

${ }^{1}$ Para la ruta A-B, los resultados fueron chi-cuadrado .789, $\mathrm{p}>0.05$, mientras que para la ruta B-A los resultados fueron chi-cuadrado $.001, \mathrm{p}>0.05$. Para la ruta $\mathrm{X}-\mathrm{Y}, \mathrm{los}$ resultados fueron chi- cuadrado $.00, \mathrm{p}>0.05$, mientras que para el segmento $\mathrm{Y}-\mathrm{X}$, se detectó un chi-cuadrado 2.04, $\mathrm{p}>0.05$ ) 
Rodrigo Mora

\section{REFERENCIAS}

ANTES, J. R.; MCBRIDE, R.; COLLINS, J. D. The effect of a new city traffic route on the cognitive map of its residents. Environment and Behavior, [S.1.], v. 20, n. 1, p. 75-91, 1988.

BAILENSON J., SHUM M., UTTAL D. The initial segment strategy: a heuristic form route selection. Memory and Cognition, [S.1.], v. 28, n. 2, p. 306-319, 2000 .

CANTER, D.; KAGG, S. Distance estimation in cities. Environment and Behavior, [S.1.], v. 7, n. 1, p. 59-79, 1975.

CLARK, A. Being there: putting brain, body and world together again. Cambridge, MA: The MIT Press, 1997.

CLARK, A. Supersizing the mind. Oxford: Oxford University Press, 2008.

CROMPTON, A. Perceived distance in the city as a function of time. Environment and Behavior, [S.1.], v. 38, n. 2, p. 173-182, 2006.

CONROY-DALTON, R. The secret is to follow your nose: route path selection and angularity. Environment and Behavior, [S.1.], v. 35, n. 1, p. 107-131, 2003.

GOLLEDGE, R. G. et al. A conceptual model and empirical analysis of children's acquisition of spatial knowledge. Journal of Environmental Psychology, [S.1.], v. 5, p. 125-152, 1985.

GOLLEDGE, R.; STIMSON R. J. Spatial behavior: a geographic perspective. New York: Guilford. 1997.

GOLLEDGE, R. G. Path selection and human preference in human navigation: a progress report. In: CONFERENCE ON INFORMATION THEORY, COSIT, 2 ., 1995, Semmering, Austria. Proceedings... Lecture Notes in Computer Science, [S.1.], v. 988, p. 207-222, 1995.

HILLIER, B.; IIDA, S. Network and psychological effects in human movement. In: CONFERENCE ON INFORMATION THEORY, COSIT, 7., 2005, Ellicottville, NY. Proceedings... Lecture Notes in Computer Science, [S.1.], v. 3693, p. 475490, 2005.

HOCHMAIR, H. Investigation of preference between the least-angle strategy and the initial segment strategy for route selection in unknown environments. Environment and Planning B: Planning and Design, [S.1.], v. 32, p. 673-691, 2005. 
JANZEN, G. et al. Oblique angled intersections and barriers: navigation through a virtual maze. In: SPATIAL COGNITION, 2., 2000, Berlin. Proceedings... Lecture Notes in Computer Science, [S.1.], v. 1849, p. 227-294, Springer, 2000.

KIRTLAND, K. A. et al. Environmental measures of physical activity supports: perception versus reality. American Journal of Preventive Medicine, [S.1.], v. 24, n. 4 , p. 323-331, 2003.

KLIPPEL,A.; MONTELLO, D. R. On the robustness of mental conceptualizations of turn direction concepts. In: INTERNATIONAL CONFERENCE ON GEOGRAPHIC INFORMATION SCIENCE, 3., 2004, Adelphi, Maryland. Proceedings...:Maryland: University of Maryland, 2004, p. 139-141.

LEISER, D.; ZILBERSHATZ, A. The traveler: a computational model of spatial network learning. Environment and Behavior, [S.1.], v. 21, n. 4, p. 401-425, 1989.

MCCORMACK G. et al. Objective versus perceived walking distances to destination. Environment and Behavior, [S.1.], v. 30, n. 3, p. 435-463, 2008.

MONTELLO, D. R. The measurement of cognitive distance: methods and construct validity. Journal of Environmental Psychology, [S.1.], v. 11, p. 101-122, 1991.

NOË, A. Action in Perception. Cambridge, MA: The MIT Press, 2004.

PEPONIS, J.; ZIMRING, C.; YOON, K. C. Finding the building in wayfinding. Environment and Behavior, [S.1.], v. 22, n. 5, p. 555-590, 1990.

PERUCH, P., GIRAUDO D. GÄRLING T. Distance cognition by taxi drivers and the general public. Journal of Environmental Psychology, [S.1.], v. 9, p. 233-239, 1989.

SADALLA, E.; MAGEL, S. The perception of traversed distance. Environment and Behavior, [S.1.], v. 12, n. 1, p. 65-79, 1980

SADALLA, E.; STAPLIN, L. The perception of traversed distance: intersections. Environment and Behavior, [S.1.], v. 12, n. 1, p. 167-182, 1980.

SADALLA, E.; STAPLIN, L.; BURROUGHS, W. Retrieval processes in distance cognition. Memory and Cognition, v. 7, n. 4, p. 291-296, 1979.

THOMPSON, E. Mind in life: biology, phenomenology, and the sciences of mind. Cambridge, MA: The MIT Press, 2007. 


\section{Rodrigo Mora}

TVERSKY, B. Structures of mental spaces: how people think about space. Environmental and Behavior, [S.1.], v. 35, n. 1, p. 66-80, 2003.

VARELA, F.; THOMPSON, E.; ROSCH, E. The embodied mind. Cambridge, MA: The MIT Press, 2003.

Recibido em: março de 2010

Aceito em: agosto de 2010 